\title{
Transversal Pressure Effect in Circulative Separators
}

\author{
Jerzy M. Sawicki \\ Gdańsk University of Technology, Faculty of Civil and Environmental Engineering, ul. Narutowicza 11/12, \\ 80-233 Gdańsk, Poland, e-mail: jsaw @ pg.gda.pl
}

(Received September 13, 2011; revised October 28, 2011)

\begin{abstract}
Progressive urban development of the human environment requires new methods of rain water treatment. Recently, there has been a growing interest in the improvement of gravitational suspension separation, and especially in the application of the centrifugal force. This important factor can be induced in two ways; by the circulation of the reservoir containing the fluid (centrifugal separators), or by a tangent supply of this reservoir (circulative separators). In addition to the centrifugal force, another essential transversal force is at work in this case, resulting from the local variability of the pressure. In the literature, this force is derived for centrifuge conditions, but applied also to circulative separators, which is questionable, as in the latter devices velocity and pressure fields are clearly different. The paper is devoted to the determination of the transversal pressure effect in circulative separators. First, a model of tangent and radial velocity profiles is introduced. The radial pressure distribution, calculated on this basis and verified experimentally, leads to the final result, that is, a technical formula describing the force in question.
\end{abstract}

Key words: centrifugal force, cyclones, rotational separators, sedimentation, waste water treatment

\section{Notation}

The following symbols are used in this paper:
A - Archimedes force,
$B-$ coefficient,
$d_{\text {in }}-$ inlet diameter,
$E \quad-\quad$ kinetic energy,
$F_{C}-$ centrifugal force,
g - gravitational acceleration,
$H \quad-$ depth,
$l_{m} \quad-\quad$ mixing length,
$m_{P} \quad$ - particle mass,
n - unit normal vector,
$p \quad-$ pressure, 


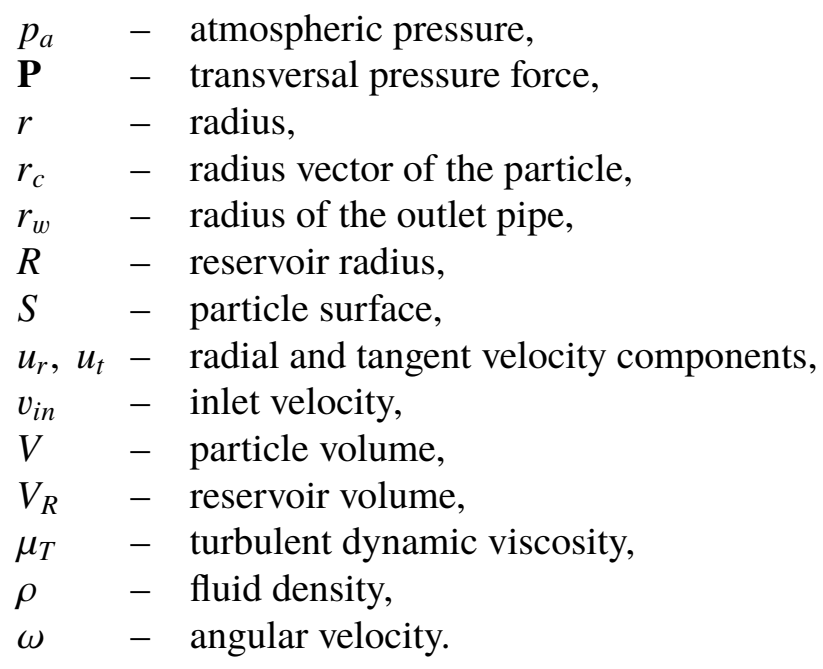

\section{Introduction}

\subsection{General Remarks}

Each curvilinear motion generates the centrifugal force:

$$
F_{c}(r)=m_{p} \omega^{2} r,
$$

where: $r$ - radius of particle trajectory curvature; $m_{p}$ - particle mass, $\omega$ - angular velocity. The centrifugal force favours the separation of suspended particles. This effect has been applied in practice for a long time, especially in the construction of gravitational separators. Because of different ways of inducing this force, all vortex separators can be divided into two classes:

- centrifugal separators (when rotation is induced by the circulation of the chamber, usually cylindrical);

- circulative separators (when rotation is induced by a tangential position of the inlet or by special guide vanes); these can be further divided into

- cyclones (Fig. 1 - well-known since the late 19th century; geometrically "slender", with a long conical section; especially often used for air de-dusting (Trawinski 1969));

- rotational separators (Fig. 2 - geometrically "thickset"; initially applied as de-sanders, e.g. Geiger construction (Imhoff, Imhoff 1979)).

From the technical point of view, cyclones are well-known industrial products, unlike rotational separators, which are still a relative novelty. Although the literature provides a considerable number of investigation results, beginning with Smith's classical thesis (Smith 1959) up to complex numerical simulations (e.g. Martignoni et al 2007), it is interesting that the proposed methods of desiging both devices still 


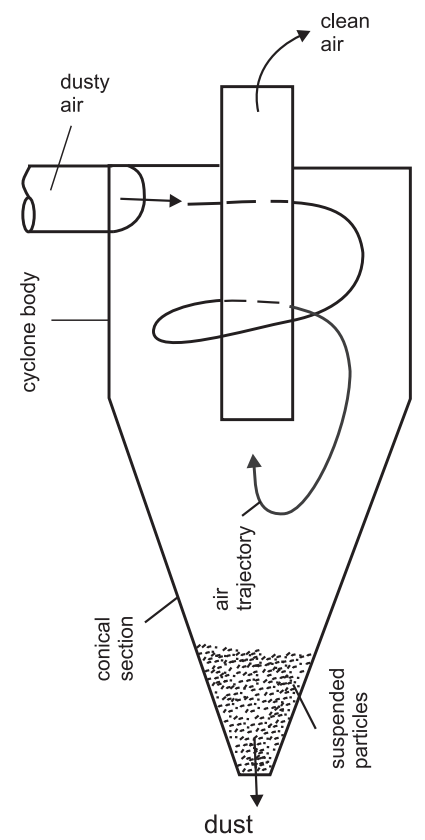

Fig. 1. Schematic axial cross-section of the cyclone

a)
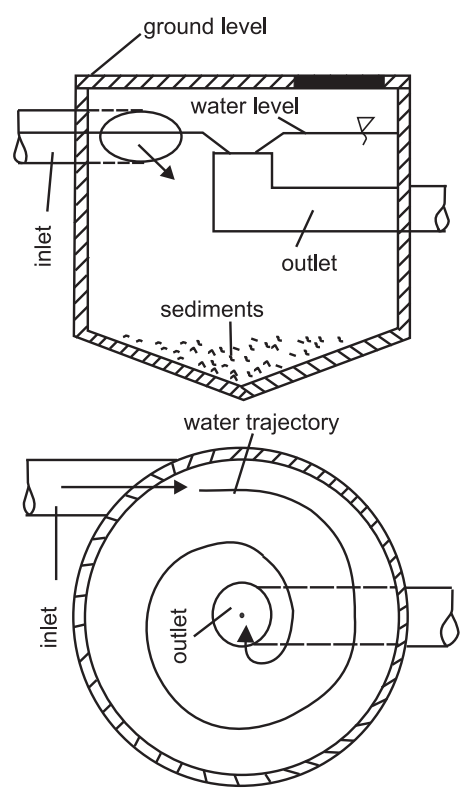

Fig. 2. Typical scheme of the rotational separator ( $\mathrm{a}$ - axial cross-section, $\mathrm{b}-$ plan view) 
raise some important doubts. The most intriguing question concerns the variability of pressure around a suspended particle.

\subsection{Transversal Pressure Effect}

Transversal pressure effect denotes the force $\mathbf{P}$, generated by the pressure field. In a general case we have (the first integral results from the definition, and the second is a consequence of Green's theorem (Beckenbach 1961):

$$
\mathbf{P}=-\int_{S} p \mathbf{n} d S=-\int_{V} \operatorname{grad} p d V,
$$

where: $p$ - pressure; $V$ - particle volume; $S$ - particle surface; $\mathbf{n}$ - unit vector, normal to $S$.

When the fluid flows along a straight line (or an almost straight line, in technical approximation), the pressure in the vertical direction varies because of gravity. In this case, the force $\mathbf{P}$ takes the form of the hydrostatic lift $\mathbf{A}$ (Archimedes force) (Landau, Lifshitz 1987):

$$
\mathbf{P}=\mathbf{A}=-\rho V \mathbf{g},
$$

where: $\rho$ - fluid density; $\mathbf{g}$ - gravitational acceleration. However, if the curvature of the fluid trajectory cannot be neglected, the pressure distribution becomes more complex, which generates an additional transversal force. This factor depends on the velocity field.

\subsection{Pressure Effect in Centrifugal Separators}

Determination of the velocity field and pressure distribution in a rotating cylinder is a classical task of hydromechanics, presented in nearly every textbook on the subject (e.g. Landau, Lifshitz 1987, Soo 1966). In this case the velocity profile has a linear character (Fig. 3a):

$$
u_{r}=0, u_{t}=\omega r,
$$

where: $u_{r}, u_{t}$ - radial and tangent components of the velocity vector. Then, the equation of fluid motion takes a very simple form:

$$
-\rho u_{r} \frac{\partial u_{r}}{\partial r}-\frac{\rho u_{t}^{2}}{r}=-\frac{\partial p}{\partial r}
$$

which yields $(C-$ integration constant):

$$
p=\frac{1}{2} \rho \omega^{2} r^{2}+C .
$$

Substituting Eq. (6) into Eq. (2), one obtains a formula describing the transversal pressure force, which acts in the centrifugal separator along the radial direction:

$$
P_{c r}=\rho \omega^{2} r_{c} V,
$$


where: $r_{c}$ - radius vector of a particle.
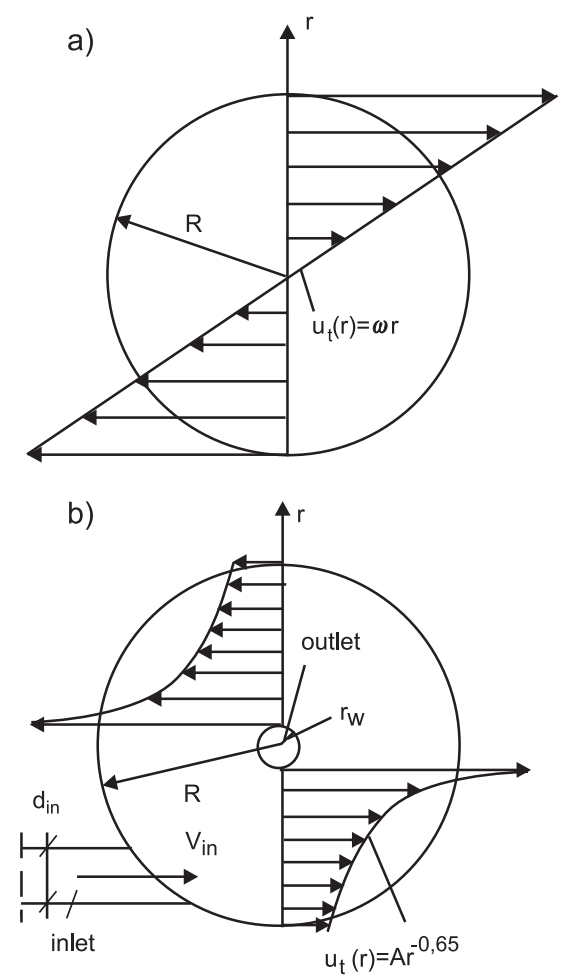

Fig. 3. Characteristic velocity profiles in vortex separators ( $a-$ centrifuge, $b$ - rotational separator)

\section{Transversal Pressure Effect}

\subsection{Velocity Field in the Circulative Separator}

It has been known for a long time that the tangent velocity field in the circulative separator differs radically from the velocity distribution in the centrifuge (Fig. 3). In the first case the maximal value of $u_{t}$ occurs near the central pipe, for $r=r_{w}$, whereas the minimal near the outer wall, for $r=R$ (Slattery 1999). Moreover, the radial velocity component $u_{r}(r)$ must also be taken into account. This means that the pressure profiles for these two kinds of devices must be different.

Despite this evident conclusion, even some of popular and practically important literature (e.g. Wikipedia 2010), describes the transversal pressure force by the same expression (Eq. 7), derived for Eq. (6), for both centrifuges and circulative separators.

To put an end to this misunderstanding, one should first determine the tangent velocity field in the circulative separator. A model of this variable should be precise, but formally simple as well. The results of Stairmand's (Slattery 1999) investigations 
(Fig. 3b) and the continuity equation indicate that the relations can be expressed by the following functions:

$$
u_{t}(r)=B r^{-0.65}, u_{r}(r)=\frac{Q}{2 \Pi H r},
$$

(note: this model does not take into account the fact that velocity drops to zero near the boundary layer, for $r=r_{w}$ and $r=R$, as this drop is a very local effect)

The multiplier $B$ can be calculated from the physically evident condition that for the steady states (which are characteristic of technical applications) the flux of mechanical energy, delivered to the object by the inflowing fluid $E_{\text {in }}$ must be equal to the flux of energy dissipated during the circulation $E_{d i s}$. This means that the energy balance of the flow can be expressed as a sum of two terms. The first describes the angular motion, supplied with energy by the inflowing stream. This stream is specially shaped (tangent to the wall) to induce the circulation. One has to underline that for a different position of the inlet its kinematic energy would be dissipated by the flow itself (in some cases this process is induced deliberately, for example by special baffles or deflectors). The second component of this energy balance takes into account the energy of the radial flow, generated by the pressure drop (in pressure reservoirs discussed in this paper) or by the free-surface inclination (in open reservoirs). This duality of the energy balance can be easily observed in fluid-flow reactors (especially in sewage treatment plants). Formally, the energy of the inlet can be passed to the outlet, but this undesirable phenomenon ("short circuit") is not a subject of this paper.

From the definition (Fig. 3b) we have ( $Q$ - discharge of water):

$$
E_{i n}=\frac{1}{2} \rho Q v_{i n}^{2}=\frac{8 \rho Q^{3}}{\pi^{2} d_{i n}^{4}} .
$$

The value $E_{d i s}$ can be calculated from the relation (Slattery 1999)

$$
E_{d i s}=\frac{1}{2} \int_{V_{r}} \mu_{T} r^{2}\left[\frac{\partial}{\partial r}\left(\frac{u_{t}}{r}\right)\right]^{2} d V_{R},
$$

where: $V_{R}$ - reservoir volume.

The coefficient of turbulent viscosity $\mu_{T}$ can be expressed by the convenient algebraic relation (Launder, Spalding 1972):

$$
\mu_{T}=0.00113 \rho u_{t}(r) l_{m}(r),
$$

with the mixing length described by Prandtl's classical concept:

$$
l_{m}=0.41 r\left(\text { for } r<\frac{R}{2}\right) l_{m}=0.41(R-r)\left(\text { for } r>\frac{R}{2}\right) .
$$


Substitution of the last three formulas into Eq. (10) yields:

$$
E_{d i s}=0.00387 H \rho B^{3}\left[\frac{1}{r_{w}^{0.95}}-\frac{1.41}{R^{0.95}}\right] .
$$

Comparing Eqs. 9 and 13, one obtains:

$$
B=4.63 Q\left[H d_{i n}^{4}\left(\frac{1}{r_{w}^{0.95}}-\frac{1.41}{R^{0.95}}\right)\right]^{-1 / 3} .
$$

One should note that the unit of this semi-empirical multiplier, namely $[B]=\mathrm{m}^{1.65} / \mathrm{s}$, results from Eq. (8).

\subsection{Theoretical Calculation of Radial Pressure Distribution}

This function can be determined analogically to the previous case. Substituting Eqs. 8 (where $B$ is defined by Eq. (14)) into Eq. (5), one obtains (boundary condition: $p=p_{a}$ for $r=r_{w}$ ):

$$
p(r)=p_{a}+0.77 \rho B^{2}\left(\frac{1}{r_{w}^{1.3}}-\frac{1}{r^{1.3}}\right)+\frac{\rho Q^{2}}{8 \Pi^{2} H^{2}}\left(\frac{1}{r_{w}^{2}}-\frac{1}{r^{2}}\right) .
$$

\subsection{Experimental Verification of Radial Pressure Distribution}

In order to verify Eq. (15), a laboratory model of a closed circulative reservoir has been built. The model has a cylindrical shape and works under pressure (Fig. 4). The pressure values were measured at three test points (A, B and C) for different intensities of the flow $Q$ with a spring-type manometer (model 611.10.063, by WIKA Manometer AG, Switzerland, class 1.6, measuring range: 0-40 mbar, accuracy of measurement: 1 mbar).

Sample results of these measurements are shown in Fig. 5, compared with overpressure $p(r)-p_{a}$, calculated from Eq. (15). The agreement of theoretically predicted and experimentally obtained results confirms the validity of the model proposed in this paper (Eqs. 8, 15).

\section{Results}

Substituting Eq. (15) into Eq. (2), one can calculate the force $P_{R r}$, acting along the radius of the circulative separator. Defining the limits of integration according to Fig. 6 and taking into account that:

$$
d V=S(r) d r
$$



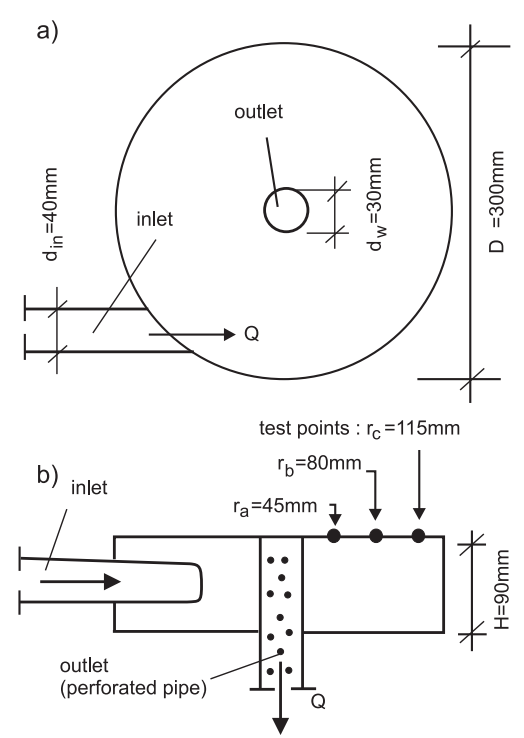

Fig. 4. Laboratory model of the circulative separator

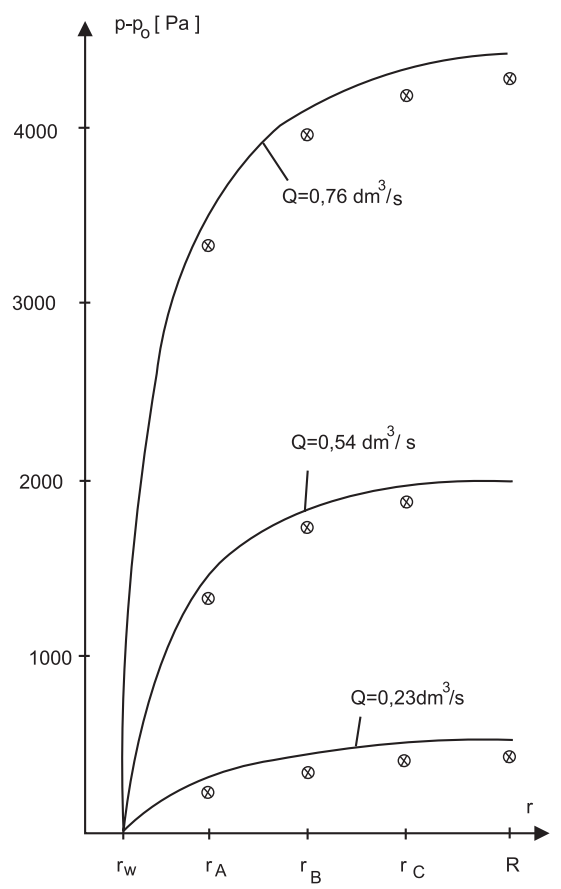

Fig. 5. Comparison of theoretical and experimental overpressure in the circulative separator 


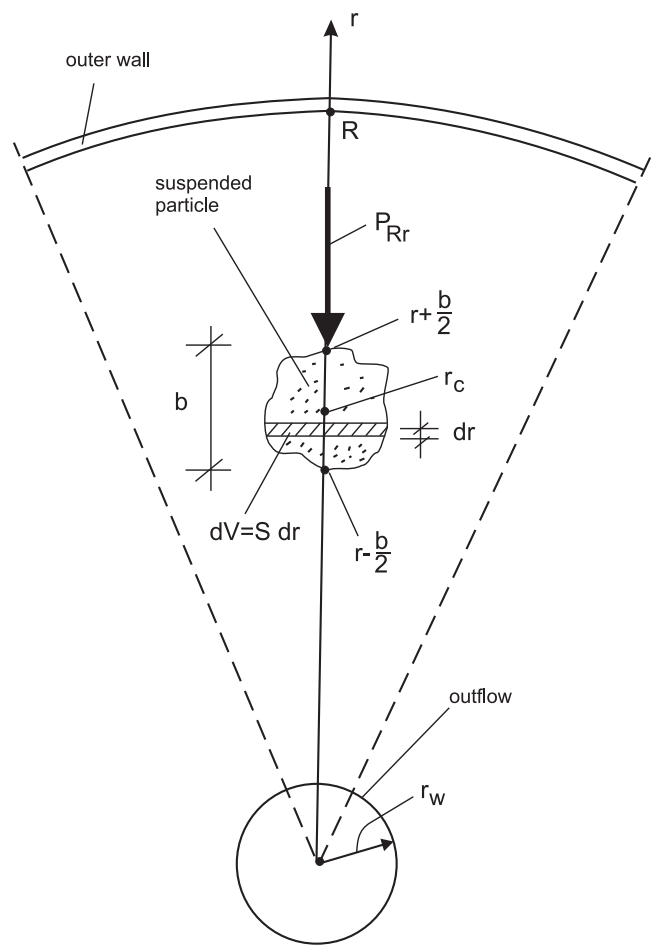

Fig. 6. Calculation of the transversal pressure effect

we can express this force as follows:

$$
P_{R r}=-0.77 \rho B^{2} \int_{r-b / 2}^{r+b / 2} \frac{\partial}{\partial r}\left(\frac{1}{r_{w}^{1.3}}-\frac{1}{r^{1.3}}\right) S d r-\frac{\rho Q^{2}}{4 \Pi^{2} H^{2}} \int_{r-b / 2}^{r+b / 2} \frac{\partial}{\partial r}\left(\frac{1}{r_{w}^{2}}-\frac{1}{r^{2}}\right) S d r .
$$

After performing all necessary calculations, one obtains:

$$
P_{R r}=-0.77 \rho V B^{2} r_{c}^{-2.3}-\frac{\rho Q^{2} V}{\left(8 \Pi^{2} H^{2} r_{c}^{3}\right)}
$$

The negative sign in this expression means that the transversal pressure force acts from the outer wall towards the center of the reservoir.

The same result could be obtained by replacing the more general relation (2) with the formula derived in the book Soo (1966):

$$
P_{R r}=-V \frac{\partial p}{\partial r}
$$




\section{Discussion}

In both centrifuges and circulative separators, the centrifugal force is a very important factor, which supports the process of the gravitational removal of suspension. However one should note some important differences between these two kinds of devices, which result from their different velocity and pressure profiles.

In the centrifuge, the angular velocity is constant, which means that the highest values of the centrifugal force and the transversal pressure effect (given by Eq. (7)) appear near the outer wall.

In circulative separators conditions are radically different. Because of a curvilinear velocity profile (Stairmand 1951), a non-zero radial velocity and specific pressure distribution (Eq. 15), the maximal values of these forces occur in the neighborhood of the central pipe.

This important fact must be taken into account while desiginig the devices discussed here.

\section{Acknowledgement}

This research has been carried out as part of the project "Innovative resources and effective methods of improving the safety and durability of buildings and transport infrastructure in the sustainable development", financed by the European Union from the European Fund for Regional Development under the Operational Programme "Innovative Economy".

\section{References}

Beckenbach E. F. (1961) Modern Mathematics for the Engineer, McGraw Hill Book Comp., New York. Imhoff K., Imhoff K. R. (1979) Taschenbuch der Stadtentwaeserung, R. Oldenburg Verlag, Vienna.

Landau L. D., Lifshitz E. M. (1987) Fluid Mechanics, Pergamon, Elmsford (NY).

Launder B. E., Spalding D. B. (1972) Lectures in Mathematical Models of Turbulence, Academic Press, London.

Martignoni W. P., Bernardo S., Quintani C. L. (2007) Evaluation of Cyclone Geometry and its Influence on Performance Parameters by CFD, Braz. Jour. Chem. Eng., 24, 1-17.

Slattery J. C. (1999) Advanced Transport Phenomena, University Press, Cambridge.

Smith J. L. (1959) Experimental and Analytical Study of the Vortex in the Cyclone Separator, MIT, Cambridge.

Soo L. S. (1966) Fluid Dynamics of Multiphase Systems, Blaisdell Publ. Comp., London.

Stairmand C. J. (1951) The Design and Performance of Cyclone Separators, Trans. Inst. Chem. Eng., 29, 356-373.

Trawinski H. (1969) Practical Aspects of the Design and Industrial Applications of the Hydrocyclones, Filtr. and Sep., 6, 361-367.

Wikipedia, the Free Encyclopedia (2010) Cyclonic Separation, http://en.wikipedia.org/wiki/Cyclonic-separation. 